\title{
Prevalencia del Intervalo QT Prolongado en diferentes patrones electrocardiográficos. Estudio por Teleelectrocardiografía
}

\author{
Drs., Mirta.Orellana A., Francesca.Bello C., Edgardo Escobar C., Patricia Adriazola G., \\ Pamela Trejo P., Interno Pablo González O. \\ ITMS, Telemedicina de Chile
}

\section{Resumen}

Introducción: El intervalo QT prolongado ha sido identificado como factor de riesgo de arritmias ventriculares incluso en personas aparentemente sanas.

El objetivo de este estudio fue establecer la prevalencia de QTC prolongado en electrocardiogramas normales, con bloqueo completo de rama (BCR) y con crecimiento de ventrículo izquierdo (HVI).

Métodos y Resultados: Se consideró como prolongado un valor $>440$ mseg, en cada una de 4 derivaciones: D1, aVL, V5 y V6, consensuado por dos observadores. El grupo 1 estuvo formado por 8.459 trazados normales: de ellos un $84 \%$ tenían un QTC normal y un 16\% tenían un QTC prolongado. El grupo 2 consistió en 2.647 trazados con bloqueo completo de rama, 532 trazados con bloqueo completo de rama izquierda (BCRI) y 2.115 con bloqueo completo de rama derecha (BCRD). De los BCRI un $51,5 \%$ de los trazados tenían QTc prolongado, lo cual observamos sólo en un $25 \%$ de los trazados con BCRD. El grupo 3 estuvo conformado por 2.503 trazados con HVI, 377 por criterio de voltaje (grupo 3a), 1.083 por criterio de voltaje y alteración de repolarización (grupo 3b) y 1.043 HVI sólo por criterio de alteración de repolarización (grupo 3c). Un 28\% de las HVI por criterio de voltaje (grupo 3a) y un $29 \%$ de HVI por alteración de repolarización (grupo 3c) tenían un QTC prolongado. De las HVI por voltaje y alteración de repolarización (grupo $3 b$ ) un $42 \%$ tenían QTC prolongado. De las HVI con QTC largo, un $7.6 \%$ tenian $Q T C \geq 500 \mathrm{mseg}$. Al desglosar las HVI por grupos el grupo 3a tenía 4,7 \% de los trazados con QTc largo $\geq 500$ mseg, el grupo $3 b$ un 7,9\% y el grupo 3c, un $8.3 \%$.

Conclusiones: Se concluye la alta prevalencia de QTC prolongado incluso en ECG con morfología normal, pero especialmente en BCRI y en HVI por voltaje más alteraciones de repolarización. La prolongación de QTc en BCR no mostró correlación con la duración de la depolarización. El impacto pronóstico de BCR con y $\sin$ QTc prolongado debe ser estudiado.

Palabras Claves: Telemedicina, tele-electrocardiografía, QTc prolongado.

\section{Increased Q-T interval in different electrocardiographic patterns: a remote recording electrocardiographic study}

Background. Increased Q-T interval has been identified as a risk factor for ventricular arrhythmia including normal subjects.

Aim. To establish the prevalence of an increased QTc in normal electrocardiograms $(N)$ an in those with complete bundle branch block (RBBB or $L B B B$ ) or left ventricular hypertrophy $(L V H)$.

Correspondencia: Dra. Mirta Orellana A.

Callao 3341,

Fax 4374960

Correo Eléctronico: Mioa25@hotmail.com 
M. Orellana A., F. Bello C., E. Escobar C., P. Adriazola G., P. Trejo P., Interno P. González O.

Method. D1, aVL, V5 and V6 leads were analyzed; a > 440ms QTC agreed upon by 2 observers was considered prolonged.

Results: an increased QTC was observed in $16 \%$ of $8459 \mathrm{~N}$ subjects, $51.5 \%$ of subjects with $L B B B$ and $25 \%$ of those with RBBB. Among subjects with $\angle V H, 28 \%$ of those with voltage only criteria for $L V H$ and $29 \%$ of those with ST-T changes but no voltage criteria had increased QTC. In contrast, $42 \%$ of patients with $L V H$ exhibiting both diagnostic criteria had increased QTC. In 7.6\% of LVH subjects with increased QTC, this interval was $>500 \mathrm{~ms}$, which broke down as follows: $L V H$ by voltage criteria alone $4.7 \%$, ST-T changes alone $7.9 \%$ and LVH with both voltage and ST-T criteria $8.3 \%$. In patients with complete bundle branch block, the duration of the QRS interval was not related to QTC.

Conclusion. A prolonged QTC is a frequent finding in normal ECGs, much more so in subjects with LVH associated to voltage and ST-T criteria. The prognostic impact of a prolonged QTC in patients with complete bundle branch block deserves further study.

Keywords: Increased Q-T interval, left ventricular hypertrophy, bundle branch block

Recibido el 05 de enero de 2009, aceptado el 20 de noviembre de 2009

Rev Chil Cardiol 2009; 28: 349-356

\section{Introducción}

Pese a que el intervalo QT prolongado se ha identificado como factor de riesgo de arritmias ventriculares, potencialmente fatales (torsión de las puntas), en pacientes post-infarto del miocardio, diabéticos e incluso en personas aparentemente normales $1,2,3$, un número importante de médicos no lo saben medir y sólo el $36 \%$ lo hace en forma correcta 4 .

Las causas de la prolongación del intervalo QT puede ser congénitas (variedades LQT 1, LQT 2 , LQT 3) o adquiridas, siendo factores predisponentes la edad avanzada, el sexo femenino, la hipertrofia ventricular izquierda, isquemia, hipokalemia, hipomagnesemia, bradicardia, fracción de eyección disminuida. También son numerosas las drogas que pueden inducir la prolongación del intervalo $\mathrm{QT}^{4,5}$, lo que hay que tener en cuenta especialmente si algunas de ellas se usan en forma combinada4. En los casos de crecimiento ventricular izquierdo por criterio electrocardiográfico y lo ecocardiográfico, se ha documentado asociación "significativa", no cuantificada, con QT prolongado6, siendo ambos factores de riesgo de eventos cardiovasculares. Por otro lado la valoración de la prolongación del intervalo QT en relación a la duración del QRS en los bloqueos completos de rama ha sido motivo de discusión?.
El objetivo del presente estudio es cuantificar la prevalencia del intervalo QT prolongado en trazados electrocardiográficos normales, bloqueos completos de rama y crecimientos ventriculares izquierdos registrados en el servicio de Telemedicina de Chile.

\section{Método}

El electrocardiograma (ECG) a través de Telemedicina, se registra desde una señal trasmitida telefónicamente desde un equipo Aerotel HRS, 6.0 o Cardiette y decodificada por un software especialmente diseñado (Plataforma integrada de Telemedicina, PIT) ${ }^{8}$.

El trazado de 12 derivaciones se muestra en la pantalla del computador, siendo posible seleccionar cualquiera de las 12 derivaciones para ser desplegada en una pantalla secundaria que permite efectuar la medición manual de la duración de los intervalos a velocidades entre 25 y $100 \mathrm{~mm} / \mathrm{seg}$., movilizando cursores electrónicos que se muestran en dicha ventana. La amplitud de los complejos se puede medir a $10 \circ 20 \mathrm{~mm} / \mathrm{mv}$.

La similitud entre los trazados obtenidos por Telemedicina y equipos tradicionales ha sido corroborada por el método de Altman y Bland9.

La duración del intervalo QT fue medida en las derivaciones $\mathrm{D} 1, \mathrm{aVL}, \mathrm{V} 5$ y $\mathrm{V} 6$ por dos observadores 
para consensuar la identificación del comienzo del complejo QRS y el término de la onda T, excluyendo la onda $U$, a una velocidad de registro de $100 \mathrm{~mm} / \mathrm{seg}$. Se seleccionaron para las mediciones sólo trazados en ritmo sinusal.

El sistema corrige automáticamente por la raíz cuadrada del intervalo $R R$, (QTC), según fórmula de Bazett y Kenigsberg 10 .

Se definió como QTc prolongado un valor $>440 \mathrm{mseg}$, en cada una de las 4 derivaciones, eligiéndose para el análisis posterior el valor más prolongado.

Se estudiaron 8.459 trazados normales (grupo 1), 2.647 trazados con bloqueo de rama que correspondían a 532 bloqueos completo de rama izquierda (BCRI) y 2.115 bloqueos completo de rama derecha (BCRD) (grupo 2) y 2.503 crecimientos ventriculares izquierdos (HVI) (grupo 3).

El diagnóstico de crecimiento de ventrículo izquierdo (HVI) fue hecho usando el índice de Sokolow para HVI por criterio de voltaje (Grupo 3a); lo mismo más T negativa asimétrica para HVI por voltaje y alteraciones de la repolarización (grupo $3 b$ ) y $\mathrm{T}$ negativa asimétrica en D1, aVL, V5 y V6 para HVI sólo por alteraciones de la repolarización (grupo $3 c$ ).

\section{Resultados}

Grupo 1. De una base de datos de 142.516 ECG se eligieron 8.459 trazados totalmente normales. De ellos, $84 \%$ tenía QTc normal y $16 \%$ QTc prolongado. (Tabla 1 , figuras 1 y 2 ).

Grupo 2. De un total de 532 trazados que cumplían criterios de BCRI, un $48,5 \%$ tenía QTc normal y
51,5\% QTC prolongado. De los 2.115 trazados electrocardiográficos con BCRD, 75\% tenía QTC normal y $25 \%$ QTc prolongado (Tabla 1 , figura 1 ).

No hubo diferencias significativas en cuanto a edad en la prevalencia de QTC prolongado. Los valores promedios de QTc fueron similares en ambos grupos.

Por método de correlación de Pearson no se detectó relación entre la duración del QRS y la duración del QTC en los bloqueos de rama (Figuras 3 y 4 ).

Para descartar la influencia de la frecuencia cardíaca en estos casos se hizo el mismo estudio en BCRI y BCRD a una frecuencia fija de 82 y $77 /$ min respectivamente (moda) confirmándose la falta de correlación señalada (Figuras 5 y 6 ).

Grupo 3. 2.503 trazados electrocardiográficos cumplían con alguno de los 3 criterios diagnósticos de HVI. QTC prolongado estuvo presente en $34 \%$ de estos trazados. De 377 ECG con HVI por voltaje (grupo 3a) y de 1.043 con HVI por alteraciones de la repolarización (grupo 3c), $28 \%$ y $29 \%$ respectivamente tenían QTc prolongado. De los 1.083 ECG con HVI por voltaje y alteraciones de la repolarización (grupo 3b) $42 \%$ tenían QTc prolongado (Tabla 1, figura 2)

Los valores promedios de QTc fueron similares en los 3 grupos $(465,468$ y $466 \mathrm{mseg})$ sin diferencias significativas en hombres y mujeres. En relación a la distribución etárea el $70 \%$ de los pacientes tenían sobre 50 años de edad.

Un 7,6\% de los QTc prolongados tenían un valor $\geq 500 \mathrm{mseg}$, lo que corresponde a un $2,63 \%$ del total de las HVI (grupo 3 a,b y c).

Tabla 1: Patrón electrocardiográfico y prolongación del intervalo QTc

\begin{tabular}{|c|c|c|c|c|}
\hline & \multicolumn{2}{|c|}{ QTc Normal } & \multicolumn{2}{|c|}{ LQTC } \\
\hline & $\mathrm{N}$ & $\%$ & $\mathrm{~N}$ & $\%$ \\
\hline ECG Normales & 7.066 & $84 \%$ & 1.393 & $16 \%$ \\
\hline \multicolumn{5}{|l|}{ Bloqueos de rama } \\
\hline BCRD & 1.586 & $75 \%$ & 529 & $25 \%$ \\
\hline $\mathrm{BCRI}$ & 258 & $48,5 \%$ & 274 & $51,5 \%$ \\
\hline \multicolumn{5}{|l|}{ HVI } \\
\hline Voltaje & 271 & $72 \%$ & 106 & $28 \%$ \\
\hline Volt + repol. & 628 & $58 \%$ & 455 & $42 \%$ \\
\hline repolarización & 741 & $71 \%$ & 302 & $29 \%$ \\
\hline
\end{tabular}


M. Orellana A., F. Bello C., E. Escobar C., P. Adriazola G., P. Trejo P., Interno P. González O.

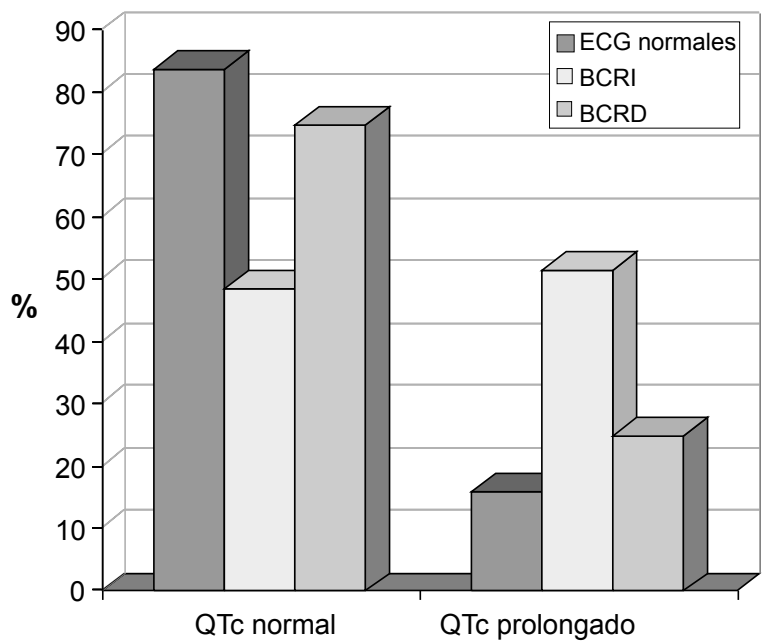

Figura 1: Distribución de QTc normal y QTc prolongado en trazados normales y bloqueos de rama.

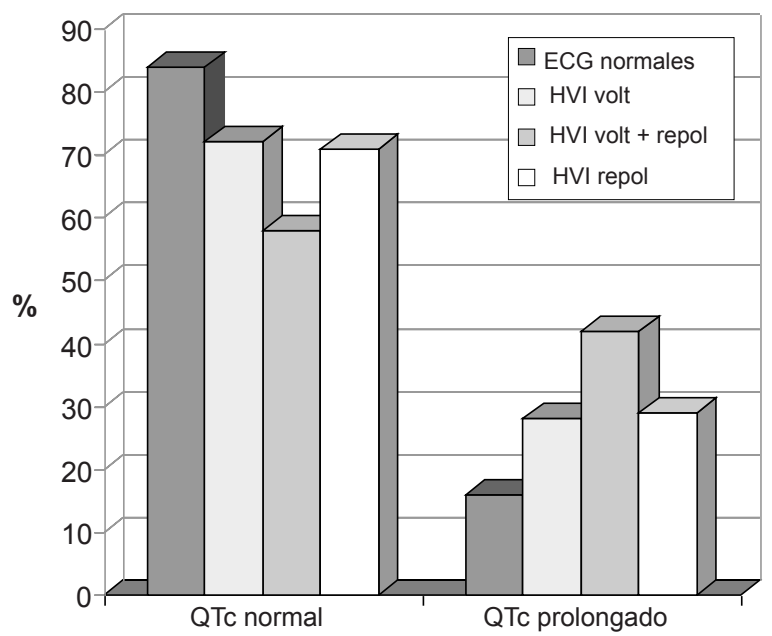

Figura 2: Distribución de QTc normal y QTc prolongado en trazados normales e hipertrofia ventricular izquierda

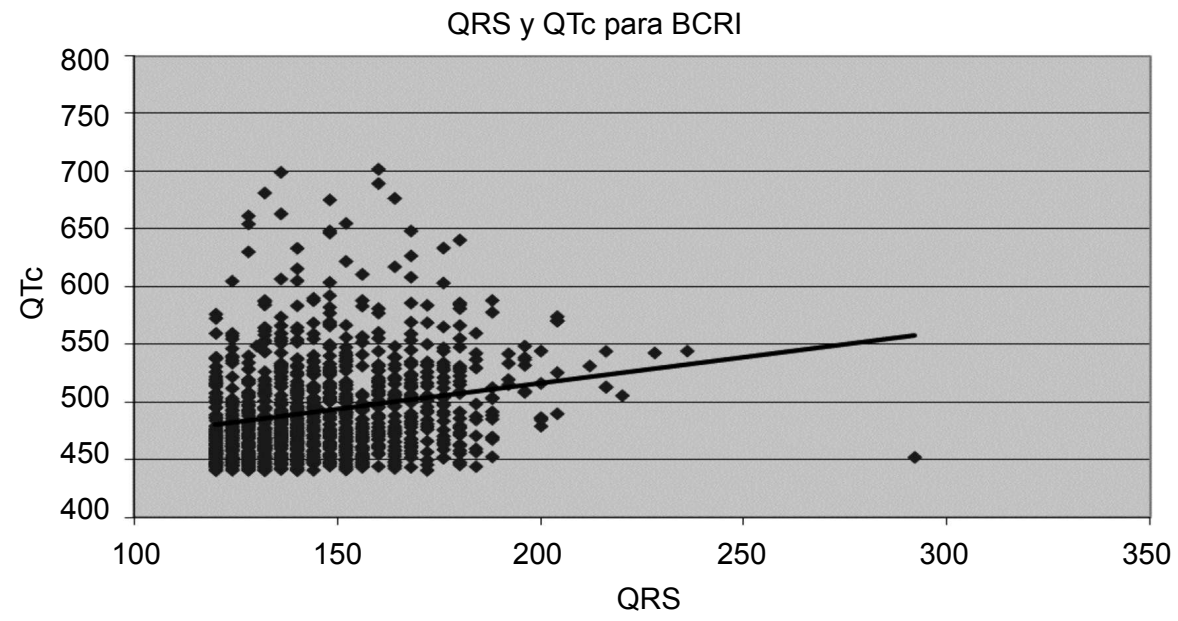

Figura 3: Correlación entre duración de QRS y QTc en bloqueo completo de rama izquierda. 


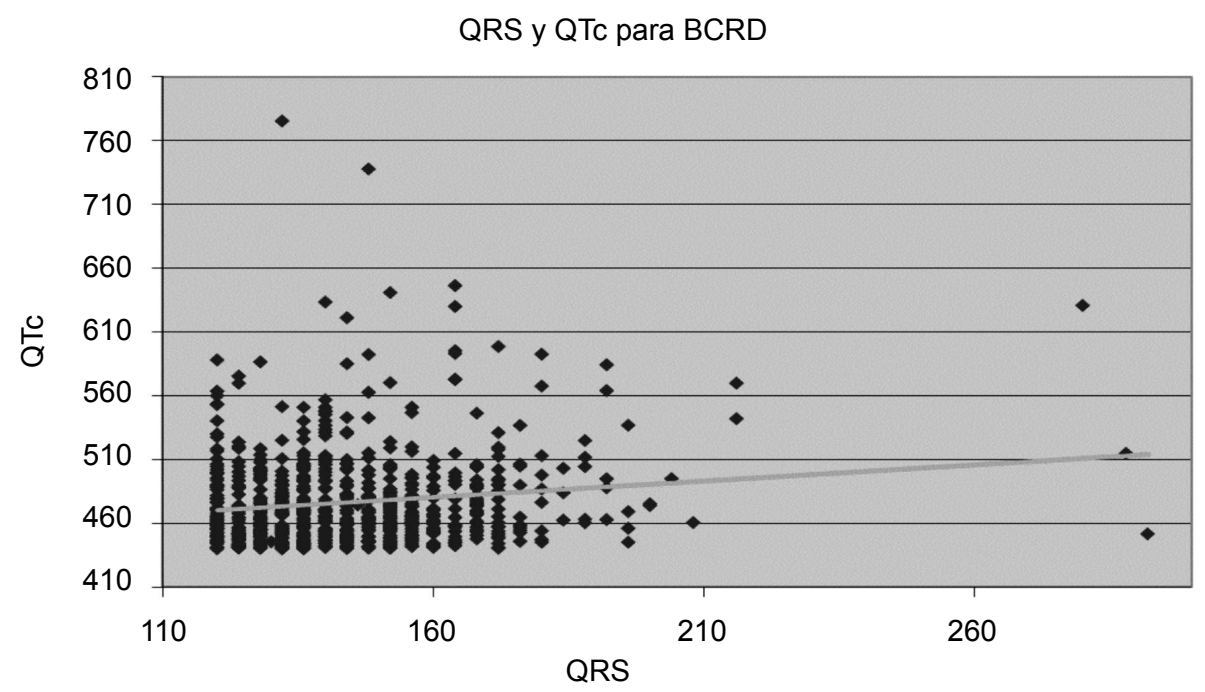

Figura 4: Correlación entre duración de QRS y QTc en bloqueo completo de rama derecha.

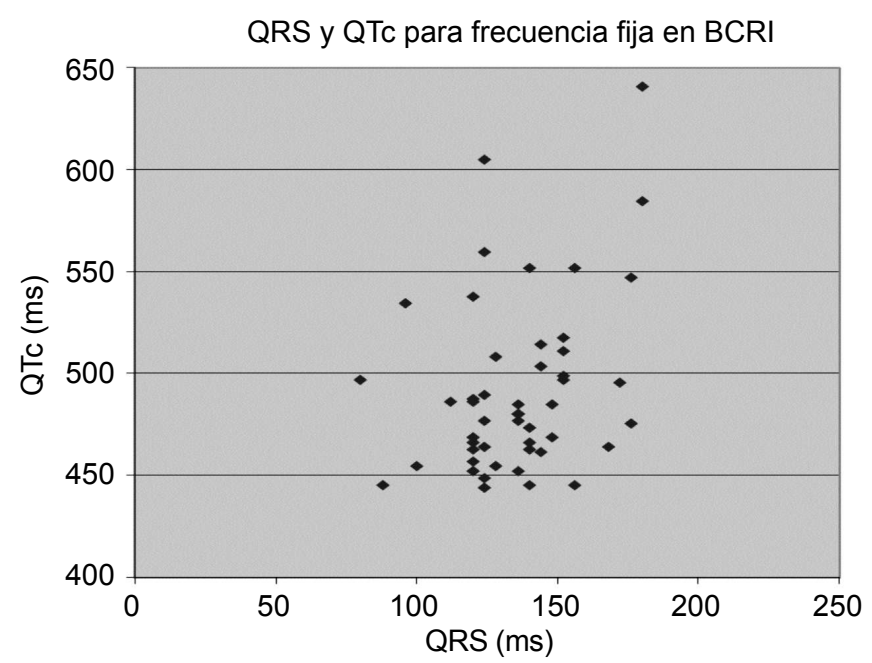

Figura 5: Duración de QRS y QTc a frecuencia cardíaca fija en bloqueo completo de rama izquierda.

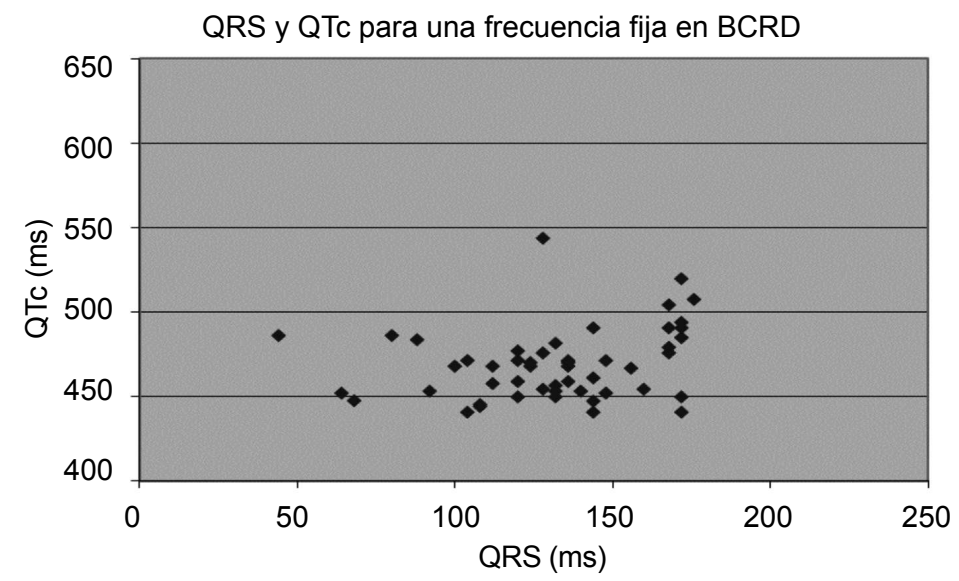

Figura 6: Duración de QRS y QTc a frecuencia cardíaca fija en bloqueo completo de rama derecha. 


\section{Discusión}

Es importante diferenciar entre el simple hallazgo de un intervalo QT prolongado y el síndrome de QT prolongado (LQTS).

Este último es un trastorno familiar, definido como una "canalopatía" que afecta a individuos con propensión al síncope, taquicardia ventricular polimórfica (torsión de las puntas), y muerte súbita por arritmia. Su prevalencia se estima en el rango de 1:5000 sujetos, aunque es considerablemente mayor considerando los casos subclínicos 11,12 .

El objetivo principal de este trabajo es llamar la atención sobre la prevalencia de un intervalo QT prolongado, dado que un número importante de médicos incluyendo cardiólogos, no lo miden en forma sistemática 0 no lo hacen correctamente 4 . No nos referimos al LQTS ya que no tenemos información completa de los síntomas ni de la historia familiar.

\section{1.- Medición y valores del intervalo QT.}

El intervalo QT debe ser medido desde el comienzo más precoz del complejo QRS al final de la onda T, en varias derivaciones y eligiendo el valor más prolongado. En este estudio elegimos las derivaciones $\mathrm{D} 1, \mathrm{VVL}, \mathrm{V} 5$ y V6 y el valor fue consensuado por dos observadores, utilizando un trazado a $100 \mathrm{~m} / \mathrm{seg}$. Esto a nuestro juicio le da validez a los valores encontrados. La fórmula de Bazett sigue siendo Standard para el uso clínico aunque puede sobrecorregir a frecuencias muy rápidas o subcorregir a frecuencias muy lentas. Nuestro trabajo excluyó trazados con frecuencias menores de 60 y mayores de 100 por min. The Internacional Conference on Harmonization of Technical Requirements for Registration of Pharmaceuticals for Human Use (ICH E-14) establece que no hay consenso en relación a la elección de valores máximos normales. Sin embargo, el riesgo de torsión de las puntas empieza alrededor de $500 \mathrm{mseg}$ y aumenta después exponencialmente ${ }^{13}$. Elegimos un valor de $440 \mathrm{mseg}$ como máximo normal14, 15, 16, 17,18, aunque más recientemente para población adulta se han considerado valores entre 430 y $450 \mathrm{mseg}$ como límites y $>450 \mathrm{mseg}$ como prolongados en el hombre y de 450 a $470 \mathrm{mseg}$ como límites y $>470 \mathrm{mseg}$ como prolongado en la mujer11,19. Además el valor $>440$ mseg debía estar presente en las 4 derivaciones estudiadas y por otro lado los valores promedios del QTc estuvieron muy por encima de este valor en los grupos estudiados.

Es importante destacar la prevalencia de QTc prolongado en un $16 \%$ de trazados de morfología normal. En estos casos sería particularmente importante recabar información sobre patología concomitante, uso de medicamentos o presencia de síntomas.

\section{2.- Bloqueos de rama e intervalo QT.}

La correlación entre los bloqueos de rama y el intervalo QT ha sido motivo de diferentes estudios. La medición del intervalo QT convencional incluye la medición de la despolarización (QRS) y de la repolarización (intervalo JT).

Das 20 comparó 72 sujetos con bloqueos con 33 sujetos sin anormalidades de la conducción intraventricular. Los valores promedios de QTC fueron significativamente más prolongados en los casos con BCRD (470 mseg) y con BCRI (489 mseg) en cada categoría secundaria a la prolongación del tiempo de despolarización y no del intervalo de repolarización que no mostró diferencias significativas.

En el presente estudio llama la atención que el intervalo QTc está prolongado sólo en el $51 \%$ de los BCRI y en el $25 \%$ de los BCRD y por otro lado no se encontró correlación entre la duración del QRS y la duración del QTc en ambos casos incluso a una frecuencia cardíaca fija.

Se ha propuesto la medición del intervalo JT o QTC- QRS como alternativa de medición de la repolarización en presencia de un QRS ancho21. QTC-QRS tendría fuerte correlación residual con la frecuencia cardíaca y no sería útil, proponiéndose fórmula JT $\sqrt{R R}$ que remueve la dependencia de la frecuencia cardíaca. Estos índices fueron estudiados en pacientes post infarto del miocardio22. Los autores concluyen que pacientes con BCRI, BCRD o bloqueo intraventricular mal definido muestran duración de repolarización más prolongada que aquellos sin estos trastornos de conducción y que $Q R \sqrt{ } R R$ y JT $\sqrt{R R}$ predicen mortalidad en estos pacientes en forma independiente y significativa. 
Breidthardt et al14 han confirmado que un QRS prolongado (sin especificarse si se trataba de BCRI o BCRD), pero no un QTC prolongado se asocia a mayor mortalidad alejada en pacientes con insuficiencia cardíaca aguda inestable.

Ambos parámetros se han asociado a una mayor mortalidad en pacientes con insuficiencia cardíaca crónica ${ }^{14}$. Por otro lado, Crow et $a^{23}$ en sujetos libres de cardiopatía coronaria encontraron que QTC y JTc no eran predictores de eventos coronarios futuros en hombres pero sí en mujeres con QRS de duración normal. En sujetos con QRS de 120 mseg o más, JTc tuvo valor predictivo mayor a QTc en hombres pero no en mujeres.

En individuos con cardiopatía coronaria el BCRI o mal definido son importantes predictores de riesgo a diferencia del BCRD23.

Sería motivo de un estudio posterior prospectivo establecer el impacto clínico de QRS prolongados con y sin QTc prolongado.

3. Crecimiento de ventrículo izquierdo e intervalo QT No hemos encontrado estudios sobre la prevalencia de intervalo QT prolongado en HVI.

De particular importancia resulta esta asociación por ser ambos por separado predictores de eventos cardiovasculares. Si bien la prevalencia de QTC prolongado es más alta en HVI por voltaje y en HVI sólo por alteraciones de la repolarización (28 y $29 \%$ respectivamente) que en el grupo de trazados normales $(16 \%)$, la mayor prevalencia se observa en la HVI por voltaje más alteraciones de la repolarización (42\%), patrón electrocardiográfico que en la mayoría de los casos traduce una sobrecarga crónica de presión de significación y que está asociada a más mal pronóstico, como fue estudiado por Levy et al24. Sin embargo, estos autores no se refieren al intervalo $Q T$ prolongado como posible mecanismo. Oikarinen et al25 confirmaron que la regresión del HVI por criterios de voltaje se asocia a un acortamiento del intervalo QT y a un impacto beneficioso sobre la incidencia de muerte brusca en estos pacientes.

\section{Fortalezas y limitaciones}

Las fortalezas de este trabajo son el elevado número de electrocardiogramas estudiados, así como la medición del intervalo QT en 4 derivaciones por dos observadores. Nuestros resultados contradicen los reportes previos sobre el valor del QTc en bloqueos intraventriculares y plantean la necesidad de un estudio prospectivo sobre el pronóstico cuando dichos bloqueos se asocian a QTc normal o prolongado. Este estudio entrega además información sobre la prevalencia del QTc prolongado en distintas formas de crecimiento ventricular izquierdo.

Las principales limitaciones son la falta de un interrogatorio previamente protocolizado que permita conocer con exactitud el uso de medicamentos, antecedentes familiares, o patologías asociadas. Por otro lado no tenemos datos de seguimiento de esta población lo que sería de gran utilidad para establecer el verdadero impacto clínico de estos hallazgos.

En conclusión el intervalo QTc debe ser cuidadosamente medido en todo electrocardiograma. $\mathrm{Si}$ se encuentra prolongado el ideal es evitar medicamentos que lo prolonguen aun más, vigilar la concentración de electrolitos y hacer un seguimiento cuidadoso.

\section{Referencias}

1. SCHOUTEN E, DEKKER J, MEPPELIK P, KOK J, VANDENBROUCKE J, POOL J.QT interval prolongation predicts cardiovascular mortality in an apparently healthy population. Circulation. 1991; 84: 1516-1523.

2. PETERS RW, BYINGTON RP, BARKER A, YUZUB S. Prognostic value of prolongedventricular repolarization following myocardial infarction: the BHAT experience: the BHAT Study Group. J. Clin. Epidemiol. 1990; 43: 167-172.

3.- ROSSING P, BREUM L, MAJOR-PEDERSEN A, SATO A,
WINDING H, PIETERSEN A, et al. Prolonged QTc interval predicts mortality in patients with Type 1 diabetes mellitus. Diabet Med. 2001; 18: 199-205.

4.- LAPOINTE A, AL-KHATIB SM, KRAMER JM, BATTLE J, CALIFF RM. Deficits in knowledge related to the QT interval that could impact patient safety. J Am Coll. Cardiol. 2002; 39: 125A.

5.- ZAREBA W. Drug induced QT prolongation: Review Article. Cardiol J. 2007; 146: 523-533. 
6.- YAN GX, RIALS SJ, WU Y, LIU T, XU X, MARINCHAK RA, et al. Ventricular hypertrophy amplifies transmural repolarization dispersion and induces early after depolarization. Am J Physiol Heart Circ Physiol. 2001; 281: 1968-1975.

7.- SANTAGELO L, AMMENDOLA E, RUSSO V, CAVALLARO C, VECCHIONE F, GAROFALO S, et al. Influence of biventricular pacing on myocardial dispersion of repolarization in dilated cardiomyopathy patients. Europace 2006; 8: 502-505.

8.- FERNANDEZ F, ESCOBAR E, ADRIAZOLA P, ARAVENA J. Technology Consideration To Reach $100 \%$ Countrywide Tele-Electrocardiography Coverage : The Chilean Experiencie .ITMS Telemedicina de Chile .Santiago.Chile. Telemedicine and e-Health. 2007; 13: 167-201.

9.- ALTMAN DG, BLAND JM. Measurement in Medicine: The analysis of method comparison studies. The Statistician 1983; 32: 307-317.

10.-BAZETT HC. An Analysis of the Time-Relation of the Electrocardiogram. Heart 1920; 7: 353-370.

11.-GOLDENBERG I, MOSS A. Long QT Syndrome. J Am Coll Cardiol. 2008; 51: 2291-2300.

12.-TAGGART NW, HAGLUND CM, TESTER DJ, ACKERMAN MJ. Diagnosis miscues in congenital long QT syndrome . Circulation 2007; 115: 2613-2620.

13- FANOE S, HVIDT C, EGE P, JENSEN GB. Syncope and QT prolongation among patients treated with methadone for heroin dependence in the city of Copenhagen. Heart 2007; 93: 1051-1055.

14.- BREIDTHARDT T, CHRIST M, MATTI M, SCHRAFL D, LAULE $K$, NOVEANU $M,$. QRS and $Q T$ interval prolongation in the prediction of long-term mortality of patients with acute destabilised heart failure. Heart 2007; 93: 1093-1097.

15- ZAREBA W, MOSS A, SCHWARTZ P, VINCENT M, ROBINSON J, PRIORI $S$, et al. Influence of genotype on the clinical course of the long-QT syndrome. $\mathrm{N}$ Engl J Med. 1998; 339: 960-965.

16.-MÖNNIG G, ECKARDT L, WEDEKIND H, HAVERKAMP W, GERSS J, MILBERG P, et al Electrocardiographic risk stratification in families with congenital long QT syndrome. Eur Heart J. 2006; 27: 2074-2080.

17. JOHN A. SUTHERLAND The EKG. Daniel K. Onion. The Little Black Book of Cardiology Phoenix Arizona. Jones and Bartlett Publisher. 2007: 33.

18.-ERIK SANDOE and BJARNE SIGURD. Fundamentals of electrocardiography Arrhythmia Diagnosis and Management . St Gallen. Fachmed AG Verlag fur Fachmedien 1984. 26

19.-VETTER V. How to make the interpretation and correctly diagnose long QT syndrome, Editorial. Ciculation 2007; 115: 2596-2597

20.-DAS G. QT Interval and repolarization time in patients with intraventricular conduction delay. J Electrocardiol. 1990; 23: 49-52.

21.-RAUTAHARJU P, ZHANG Z, PRINEAS R, HEISS G. Assessment of Prolonged QT and JT intervals in ventricular conduction defects. Am J Cardiol. 2004; 93: 1017'-1021.

22.-PIOTROWICH K, ZAREBA W, Mc NITT S, MOSS A. Repolarization duration in patients with conduction disturbances after myocardial infartion. Am J Cardiol. 2007. 15; 99: 163-168.

23.-CROW R, HANNAN P, FOLSOM A. Prognostic significance of corrected QT and corrected JT interval for incident coronary heart disease in a general population sample stratified by presence or absence of wide QRS complex. Circulation 2003; 108: 1985-1989.

24.-LEVY D, SALOMON M, .D'AGOSTINO R, BELANGER A, KANNEL W. Prognostic implications of baseline electrocardiographic features and their serial changes in subjects with left ventricular hypertrophy. Circulation 1994; 90: 1786-93.

25.- OIKARINEN L, NIEMINEN MS, VIITASALO M, TOIVONEN L, WACHTELL K, PAPADEMETRIOU $\mathrm{V}$, et al. Relation of QT interval and QT dispersion to regression of echocardiographic and electrocardiogarphic left ventricular hypertrophy in hypertensive patients: The losartan intervention for endpoint reduction (LIFE) Study. J Hypertens. 2001; 19: 1883-1891. 